BULL. AUSTRAL. MATH. SOC.

VOL. $4(1971), 361-366$.

\title{
Homomorphisms of sharply transitive projective planes
}

\author{
Don Row
}

We construct projective planes having non-degenerate homomorphic images, the homomorphisms preserving certain sharply transitive collineation groups.

Two methods of obtaining examples of homomorphisms of projective planes are available in the literature - namely those due to Tsukerman (see [8], Theorem 4) and Klingenberg [5]. The aim of this note is to construct further examples. We do this by giving a geometric structure to groups - the structure being preserved by selected group homomorphisms using a construction of difference sets due to $\mathrm{Hall}$ [1] and Hughes [2]. The projective planes obtained in this way are sharply transitivel, each having a collineation group sharply transitive both on points and on lines. This collineation group is preserved by the induced geometric homomorphism and we note that when the group is abelian this is a characterising property of the homomorphism.

Recall the geometric structure $D(G)$ which can be attached to any group $G$ having a difference set $D$ - with all elements of $G$ as points, all subsets $g D$ as lines, and incidence determined by set inclusion. Every sharply transitive plane can be represented in this way. In a suitable group $G$ we construct a difference set $D$ so that a given group epimorphism $h: G \rightarrow G^{\prime}$ induces a projective plane epimorphism $D(G) \rightarrow D^{\prime}\left(G^{\prime}\right)$, where $D^{\prime}$ is some given difference set of $G^{\prime}$. The required condition is simply given.

Received 2 December 1970.

1 two dimensional group spaces in the terminology of Karzel [4]. 361 
LEMMA. The group epimorphism $h: G \rightarrow G^{\prime}$ induces a geometric epimorphism if $h D \subseteq D^{\prime}$.

Proof. If $x \in g D$ then $h x \in h g h D \subseteq h g D^{\prime}$, thus the point and line maps $g \rightarrow h g, g D \rightarrow h g D^{\prime}$ preserve incidence. //

Some restrictions are inherent in the problem, as involutary collineations have fixed points and non-trivial homomorphisms have infinite inverse images. Thus, for example, the following result is the strongest possible for countable abelian groups containing finite partial difference sets.

PROPOSITION. If $G$ is a countably infinite group containing no elements of even order and

(a) each element of $G$ has at most finitely many square roots in G;

and if $h: G \rightarrow G^{\prime}$ is an epimorphism with infinite kernel, $G^{\prime}$ containing a difference set $D^{\prime}$, and

(b) $\left\{x^{-1} g x \mid x \in\right.$ kernel of $\left.h\right\}$ is infinite for each element $g$ not in the centre of $G$;

then any finite partial difference set $D_{1}$ satisfying $h D_{1} \subseteq D^{\prime}$ can be extended to a difference set $D$ of $G$ satisfying $h D \subseteq D^{\prime}$.

Proof. Following the procedure of Hughes [2] we well-order $G$ and, supposing $g$ to be the least element not expressible as $d_{1} d_{2}^{-1}, d_{i} \in D_{1}$ $\left(D_{1}\right.$ may be empty), we extend $D_{1}$ to $D_{2}=D_{1} \cup\{g x, x\}$ by choosing an $x \in G$ so that $D_{2}$ is a partial difference set and $h D_{2} \subseteq D^{\prime}$.

The latter condition will be satisfied for $x$ in the (infinite) inverse image of $d_{2}^{\prime}$, where $h g=d_{1}^{\prime}\left(d_{2}^{\prime}\right)^{-1}, d_{i}^{\prime} \in D^{\prime}$. As $d_{1} d_{2}^{-1}=d_{3} d_{4}^{-1} \Leftrightarrow d_{3}^{-1} d_{1}=d_{4}^{-1} d_{2}, D_{2}$ is a partial difference set if $x$ is not a solution of any of

$$
x=g d_{1}
$$




$$
\begin{gathered}
x=g^{-1} d_{1}, \\
x=g^{-2} d_{1}, \\
x=d_{1}, \\
x=d_{1} d_{2}^{-1} d_{3}, \\
x=g^{-1} d_{1} d_{2}^{-1} d_{3}, \\
\left(x d_{1}^{-1}\right)^{2}=d_{2} d_{1}^{-1}, \\
\left(x d_{1}^{-1} g\right)^{2}=d_{2} d_{1}^{-1} g, \\
\left(x d_{1}^{-1}\right)^{2}=g^{-1} d_{2} d_{1}^{-1} g, \\
x^{-1} g x=d_{1}^{-1} d_{2},
\end{gathered}
$$

where $d_{i} \in D_{1}$.

Equations (1) to (6) have only finitely many solutions, as also do equations ( 7 ) to ( 9 ) by (a). If $g$ is in the centre of $G$ then (10) becomes $g=d_{1}^{-1} d_{2}=d_{2} d_{1}^{-1}$ which has no solution by hypothesis. Hence there is an infinite choice of $x$ for $D_{2}$ to satisfy the two requirements we ask. If $g$ is not in the centre condition (b) ensures $\left\{x^{-1} g x \mid x \in\right.$ inverse image of $\left.d_{2}^{\prime}\right\}$ is infinite. So in both cases $D_{2}$ can be constructed as desired.

We repeat the procedure for the least $g$ not expressible as $d_{1}^{-1} d_{2}, d_{i} \in D_{2}$, and using an induction argument obtain a nested union $D=\bigcup_{n \geq 1} D_{n}$ of partial difference sets $D_{n}$ in $G$. Then $D$ is the required difference set. //

We give an application. 
THEOREM 1. Every finite cyclic projective plane is the homomorphic image of an infinite cyclic projective plane. I/

In particular in the additive group $Z$ of the integers $D_{1}=\{0,1,3,9,35,100,130,269,273,367,1300,3890,3900,5188$,

$11714,13003,27327,39000,130000,157$ 283, 1170013,1300001$\}$ is a partial difference set whose image under an epimorphism onto a cyclic group $Z_{13}$ of 13 elements is a difference set $D^{\prime}$. Straightforward calculations show that the minor theorem of Desargues holds for the two triangles with vertices $-1,-3,-9$ and $34,32,26$ but not for the two triangles with vertices $-1,-3,-9$ and $2,32,11705$ in any plane $D(2)$ obtained by extending $D_{1}$. Consequently $D(Z)$ is neither a Moufang plane nor non-closed and the induced epimorphism $D(Z) \rightarrow D^{\prime}\left(z_{13}\right)$ is not obtainable by presently available generalisations of the methods of Tsukerman and $\mathrm{Kl}$ ingenberg.

The Proposition can be modified in various ways - for example showing that planes other than free planes and some Desarguesian planes have infinitely many images.

THEOREM 2. There is a projective plane, which is not free and not desarguesian, having infinitely many non-degenerate and pairwise non-isomorphic homomorphic images.

Proof. Consider an infinite class of cyclic groups $Z_{n^{2}+n+1}$, each having a difference set including the identity element (existence is guaranteed by [7]). Choosing as $G$ the direct product $z \times \prod z_{n^{2}+n+I}$, and as $D_{1}$ the canonical embedding in $G$ of the partial difference set of $Z$ given above, $D$ can be constructed to simultaneously satisfy the requirements of the Proposition for all the canonical epimorphisms $G \rightarrow z_{n^{2}+n+1}$. We need only note that the intersection of any set of inverse images, one from each of these epimorphisms, is infinite and that $D_{1}$ can be extended to a partial difference set $D_{2}$ as in the proof of the Proposition by all but a finite number of elements $x$ of $G$. // In [3] Hughes raises the question of possible preservation of 
non-central collineations by homomorphisms. Applying the isomorphism theorem of [6] it is straightforward to show that each of the homomorphisms of this note preserves a sharply transitive collineation group - this being a characteristic property when the group is abelian.

THEOREM 3. A projective plane epimorphism preserves an abelian collineation group which is transitive on points if and only if it is induced by an epimorphism of that group.

Proof. Such a group is necessarily sharply transitive on both points and on lines, and if preserved, its image is abelian and transitive also.//

\section{References}

[1] Marshall Hall, Jr, "Cyclic projective planes", Duke Math. J. 14 (1947), 1079-1090.

[2] D.R. Hughes, "A note on difference sets", Proc. Amer. Math. Soc. 6 (1955), 689-692.

[3] D.R. Hughes, "On homomorphisms of projective planes", Proc. Sympos. App 2. Math. 10, 45-52 (Amer. Math. Soc., Providence, Rhode Island, 1960).

[4] Helmut Karzel, "Bericht über projektive Inzidenzgruppen", Jber. Deutsch. Math.-Verein. 67 (1964/65), 58-92.

[5] Wilhelm KIingenberg, "Projektive Geometrien mit Homomorphismus", Math. Ann. 132 (1956), 180-200.

[6] Don Row, "A homomorphism theorem for projective planes", Buzl. Austral. Math. Soc. 4 (1971), 155-158.

[7] James Singer, "A theorem in finite projective geometry and some applications to number theory", Trans. Amer. Math. Soc. 43 (1938), 377-385. 
[8] L.A. Skornyakov, "Projective planes" (Russian), Uspehi Matem. Nauk 6 (46) (1951), 112-154.

University of Tasmania, Hobart, Tasmania 